concurrent multiple partners $(\mathrm{p}<0.001)$ and use condoms inconsistently $(\mathrm{p}<0.001)$. Married men were more likely to know the HIV status of their partners $(\mathrm{p}<0.001)$ and to have disclosed to others $(p=0.020)$. Respondents who engage in intergenerational sex were more likely to have transactional sex $(p<0.001)$ and not used condom at last sex $(p=0.010)$. Transactional sex was commoner among men who practice concurrency $(\mathrm{p}<0.001)$ and did not use condom at last sex $(\mathrm{p}=0.005)$.

Conclusion The sexual behaviour of this study population closely aligns with that of men in the general population with respect to risk taking. Targeting HIV positive men for sexual behaviour change will therefore contribute towards eliminating new HIV infections.

\section{P4.100 ADOLESCENTS' QUALITATIVE DESCRIPTIONS OF THEIR ATTITUDINAL CHANGES REGARDING HYPOTHETICAL CLINICAL TRIAL PARTICIPATION}

${ }^{1}$ Susan L Rosenthal, ${ }^{1}$ Ariel M De Roche, ${ }^{1}$ Marina Catallozzi, ${ }^{2}$ Carmen Radecki Breitkopf. ${ }^{1}$ Columbia University Medical Centre, New York, USA; ${ }^{2}$ Mayo Clinic, Rochester, USA

\subsection{6/sextrans-2017-053264.595}

Introduction Little is known about how adolescents' attitudes about willingness to participate (WTP) in clinical trials change over time.

Methods Adolescents (14-17 years) were asked two times about WTP for a hypothetical microbicide safety trial a year apart. After rating their WTP at follow-up, the adolescents were asked how their thoughts about the study had changed. They were not reminded of their previous WTP response; qualitative coding of responses was blinded to their WTP.

Results The adolescents $(n=294)$ were 69\% Hispanic, 63\% female, and had a mean age at baseline of 15.5 years. Most $(60 \%)$ adolescents reported that their thoughts had stayed the same, 33\% reported a change, which might or might not be associated with a perceived change in WTP, and 7\% stated that they did not remember. Some adolescents reported understanding the study information better; either it had been explained better or they paid greater attention. This was not always associated with a perceived change in WTP. Others reported a change in the weight of the information, either for non-specific reasons or because of new experiences (I had a yeast infection and they did the whole exam thing and it wasn't that bad) or new information (in high school I've learned about it). Others attributed changes to maturation/age (I think I'm older and I can focus more) or to a personality change (e.g. less shy). Parental impact included a few who reported an influential conversation with a parent (I know more because my parents have been talking about it) or the ability to make an independent decision.

Conclusion Most adolescents did not report a change in thinking. Some did not feel they had all the information the first time; for others, their attitudes about the information changed. For a few, independence from parents led to a change. Adolescents should be given continued opportunities to participate in clinical research over time. Future research should explore ways to ensure that adolescents understand key information to make the best decision, and to balance parental guidance with autonomy.
Support: National Institutes of Health (R01HD067287); National Centre for Advancing Translational Sciences, National Institutes of Health (UL1 TR000040, UL1 TR000457)

\section{P4.101 ADOLESCENTS' AND THEIR PARENTS' ATTITUDES OVER TIME ABOUT PARENTAL INVOLVEMENT IN CLINICAL RESEARCH}

${ }^{1}$ Susan L Rosenthal, ${ }^{1}$ Ariel M De Roche, ${ }^{1}$ Marina Catallozzi, ${ }^{2}$ Carmen Radecki Breitkopf, ${ }^{3}$ Lisa S IPP, ${ }^{3}$ Jane Chang, ${ }^{1}$ Jenny K Francis, ${ }^{1}$ Christine M Mauro. ${ }^{1}$ Columbia University Medical Centre, New York, USA; ${ }^{2}$ Mayo Clinic, Rochester, USA; ${ }^{3}$ Weil Cornell Medical Centre, New York, USA

\subsection{6/sextrans-2017-053264.596}

Introduction Adolescent participation in reproductive health clinical trials requires balancing adolescent autonomy and parental involvement. Previous work indicated that adolescents and parents viewed parental involvement as having two aspects, learning information (e.g., test results, risk behaviours) and involvement in the process (e.g. accompanying them to the appointment).

Methods Adolescents (ages 14 to 17 years) and their parents were enrolled in a longitudinal study assessing willingness to participate in a hypothetical microbicide clinical trial. They were asked at baseline and at one year follow-up to respond yes/no to 9 items regarding parental involvement. At baseline, one item "asking details about the study" was subsequently dropped from analyses.

Results The adolescents $(n=254)$ were 69\% Hispanic, 65\% female, and had a mean age at baseline of 15.5 years. Factor analysis for follow-up data indicated a different factor structure. In order to understand the change, adolescent and parent data were factor analysed separately. The adolescents' factor structure indicated that all of the items loaded on one factor, with the exception of the two items regarding getting permission from parents to participate in studies. The factor structure for those that were under 18 remained different from the factor structure at baseline, implying that being a legal adult was not the cause of the change. For the parents, the factors remained fairly similar to the baseline factor structure.

Conclusion The findings suggest that although the structure of parental attitudes about involvement in research may be stable over a year's time; adolescents may over time view parental permission as a separate concept from the general role of parents in research. This view was not related to adolescents obtaining legal status to self-consent. Understanding of why/ how attitudes about parental involvement change or stay stable over time may help investigators manage expectations.

Support: National Institutes of Health (R01HD067287); National Centre for Advancing Translational Sciences, National Institutes of Health (UL1 TR000040, UL1 TR000457)

\section{P4.102 FATHER OF THE BABY'S OPINION INFLUENCES ATTITUDES ABOUT MICROBICIDES FOR BACTERIAL VAGINISIS AMONG U.S. PREGNANT WOMEN}

${ }^{1}$ Susan L Rosenthal, 'Lauren Dapena Fraiz, ${ }^{2}$ Greg D Zimet, Susan L.Rosenthal ${ }^{3} .{ }^{1}$ Columbia University Medical Centre, New York, USA; ${ }^{2}$ Indiana University, Indiana, USA; ${ }^{3}$ Columbia University, New York, USA 
Introduction Bacterial vaginosis (BV) during pregnancy is linked to preterm labour and infant morbidities. Microbicides are in development for BV management, which could benefit pregnant women.

Methods Pregnant women in the US $(n=760)$ completed an online survey of attitudes about hypothetical microbicide use for BV. Conjoint analysis measured attitudes across the following dimensions: reason for microbicide use (prevention, prevention when at high risk, or treatment), product formulation (ring or gel), father of the baby's (FOB) attitudes (would want her to use the product, would not want her to use, or would not care), and ease of use (easy to insert or requires practice). Women rated 9 scenarios, each a unique combination of attributes (fractional factorial design) on a scale ranging from $0-100$ in increments of 10 . Five additional items addressed FOB involvement in use.

Results Most women were 25-35 years old (69\%), non-Hispanic White (65\%), married/living with the FOB (90\%), and had been pregnant before $(71 \%)$. Of the 702 women with complete responses, 75 gave the same rating for all scenarios (51 rating all as 80-100), precluding them from the conjoint analysis. The highest rated scenario $(M=72)$ was: for treatment, a gel, FOB wants her to use, easy to insert. The lowest rated $(M=63)$ was: for prevention, a gel, FOB doesn't want her to use, and takes practice. Conjoint analysis $(n=627)$ showed that FOB's attitudes was most important in influencing ratings (accounting for $49 \%$ of rating variability), followed by product formulation (22\%; preference for gel), reasons for use (18\%; preference for treatment), and ease of use $(11 \%)$. Most women reported the $\mathrm{FOB}$ would assist in microbicide use by being supportive (83\%), reminding them to use it $(81 \%)$, and going to the store to get it $(77 \%)$, and applying it $(58 \%)$.

Conclusions Pregnant women are accepting of microbicide use for BV; however, the opinion of the FOB will be critical for uptake. Once a microbicide is available, strategies to enhance uptake will need to foster the FOB's involvement and support.

Support: National Institutes of Health (R33AI098654)

\section{P4.103 BRAZILIAN YOUNG KEY POPULATION REACHED BY A HIV PEER-TESTING STRATEGY}

Tainah Dourado de Miranda Lobo; Clarissa Habckost Dutra de Barros; Diego Agostinho Calixto, Ana Roberta Pati Pascom. Department of STI, AIDS and Viral Hepatitis, Secretary for Health Surveillance, Ministry of Health, Brasilia - DF, Brazil

\subsection{6/sextrans-2017-053264.598}

Introduction Worldwide, young people have become increasingly vulnerable to HIV infection, especially those belonging to the key populations - the so-called "Young Key Population" (YKP). The Brazilian epidemic is largely concentrated in key populations and, over the last ten years, the aids detection rate among young males had a significant increase. Brazil, in partnership with selected NGOs, has developed an oral fluid rapid HIV peer-testing strategy tailored to key populations, called Live Better Knowing (LBK). Our aim is to provide an overview of HIV-related indexes of YKP tested on LBK.

Methods The LBK is an initiative driven in collaboration with NGOs, delivering HIV testing and prevention strategies targeted at the key populations and young people aged 15 to 24 yo, since 2013. Trained peers accessed participants in social venues for the intervention and applied a questionnaire about their sociodemographic information and risk behaviour. Results presented here are from data obtained anonymously by the strategy, between June 2015 and October 2016.

Results Among the 26010 participants aged 15 to 24yo, $65.2 \%$ were nonwhite, $65.7 \%$ reported drug use and $13 \%$ drug use and commercial sex combined. Overall, $53.8 \%$ reported condom use at last sexual intercourse and $7.9 \%$ reported STI symptoms in the last 12 months. The general HIV prevalence found was $1.2 \%$ and its distribution among cis women, transvestites, transsexual women, transsexual men, MSM and heterosexual cis men was $0.4 \%, 5.4 \%, 4.2 \%$, $0.5 \%, 3.2 \%$, and $0.5 \%$ respectively.

Conclusion YKP constitutes a high-risk population for HIV in urgent need of responses able to tackle their vulnerabilities related to the HIV acquisition. Given the combination of drug use with sex work, comprehensive harm reduction services need to be delivered. Despite the broad condom free distribution in the country, our analyses suggest the need to impact behaviours related to its use. LBK is a strategy tailored to groups who continue to face obstacles in accessing HIV services, thus, it should be expanded in order to reach out to more people.

\section{P4.104 FREQUENCY OF HIV TESTING WITH AND WITHOUT A CLINICAL CONSULTATION AMONG MEN WHO HAVE SEX WITH MEN (MSM): A RANDOMISED CONTROLLED TRIAL}

${ }^{1}$ Tim Rh Read, 'Sandra Walker, ${ }^{1}$ Eric Chow, ${ }^{1}$ Catriona Bradshaw, ${ }^{2}$ Rebecca Wigan, ${ }^{2}$ Marcus Chen, ${ }^{1}$ Christopher Fairley. ${ }^{1}$ Monash University, Melbourne, Australia; ${ }^{2}$ Melbourne Sexual Health Centre, Melbourne, Australia

\subsection{6/sextrans-2017-053264.599}

Introduction Increasing the frequency of HIV testing among populations at risk, such as MSM, enables earlier diagnosis which maximises the preventive effect of antiretroviral therapy. The requirement to attend a clinical consultation to obtain HIV testing may act as a barrier to increasing the frequency of testing and adds to the cost of additional tests.

Methods Randomised controlled trial (RCT). MSM attending Melbourne Sexual Health Centre for HIV testing were randomised $1: 1$ to an intervention allowing HIV/syphilis testing at a network of around 300 pathology centres and in the clinic, without requiring another consultation for 12 months. Controls had to attend a consultation for each HIV test as usual. During the study, both groups received four text messages recommending frequent testing. The primary outcome was the incidence of HIV testing over 12 months.

Results Of 443 men referred to the study, 13 declined participation, 8 were excluded because they were leaving Victoria and 422 were randomised. Three had positive HIV tests and were excluded from the analysis. Of 208 controls, 202 (97.1\%) and 200 of $211(94.8 \%)$ intervention group members were followed to 12 months. The intervention group had 453 tests in 205.6 person-years, incidence rate 2.2 (95\% confidence interval (CI): $2.0,2.4)$ tests per year and the control group had 432 tests during 204.0 person-years, incidence 2.1 (95\% CI: 1.9, 2.3) tests per year, incidence rate ratio (IRR) 1.04 (95\%CI: $0.89,1.2) \mathrm{p}=0.63$. The intervention group had 101 of 453 [22.3\% (95\% CI:18.5\%, 26.4\%)] tests at pathology centres without a clinical consultation.

Conclusion In this RCT, MSM who were allowed HIV/syphilis testing outside of clinical consultations did not test more 
frequently than controls who had to attend a consultation for every test. Making testing more convenient may not be sufficient to achieve increases in testing frequency. However more than one in five tests in the intervention group did not require a clinical consultation, reducing service costs.

Trial registration: ACTRN12614000760673

\section{P4.105 HIGHLY SUCCESSFUL ENGAGEMENT IN AN ACUTE HIV- INFECTION (AHI) AWARENESS CAMPAIGN AND INTERVENTION IN AMSTERDAM \& ITS YIELD OF AHI DIAGNOSES AT THE CITY'S STI CLINIC}

${ }^{1}$ Udi Davidovich, ${ }^{2}$ Maartje Dijkstra, ${ }^{3}$ Arjan Van Bijnen, ${ }^{3}$ Sjoerd Van Elsen, ${ }^{1}$ Maarten Schim Van Der Loeff, ${ }^{4}$ Fred Verdult, ${ }^{1}$ Elske Hoornenborg, ${ }^{5}$ Godelieve De Bree, ${ }^{3}$ Wim Zuilhof. ${ }^{1}$ Amsterdam Public Health Service, Amsterdam, The Netherlands; ${ }^{2}$ Academic Medical Centre, University of Amsterdam, Amsterdam, The Netherlands; ${ }^{3}$ SOA AIDS Nederland (STI Netherlands), Amsterdam, The Netherlands; ${ }^{4}$ Volle Maan Communications Agency, Amsterdam, The Netherlands; ${ }^{5}$ The Amsterdam Institute for Global Health and Development (AlGHD), Amsterdam, The Netherlands

\subsection{6/sextrans-2017-053264.600}

Introduction Acute HIV infection (AHI) is associated with high HIV viral load and therefore an important factor in onward transmission. Timely detection and treatment of AHI can contribute to a better HIV prognosis and help prevent further transmission.

Methods A media campaign directed men to an online intervention (hebikhiv.nl) where visitors were trained in recognising AHI-symptoms and offered an interactive risk-assessment questionnaire using a risk score algorithm comprising behaviour and symptoms. If found at risk, men were referred to the Amsterdam STI clinic for AHI testing including a point-of-care HIV RNA test. If diagnosed with AHI, counselling and referral for immediate treatment was offered. Participation was monitored using web-statistics, and men presented for testing were questioned regarding their referral source.

Results From Aug 2015 to Dec 2016, the intervention's website was visited 150035 times by 127394 unique visitors. The online risk-assessment tool was started 96756 times and was completed in $81 \%(77,949)$ of the cases. Of those, $10 \%$ (7733) received the advice to test for AHI, and of those, 21\% (1609) downloaded the referral letter to the STI clinic. At the clinic, 209 men presented for AHI testing. Of those $57 \%$ (119) were confirmed to have arrived through the campaign. In total, 181 were eligible for testing, and in $7 \%$ (13) AHI was diagnosed. Of the 13 AHI cases, 2 were referred by the campaign, 5 by the STI clinic, 4 through own initiative, 1 by 'others', and none by a GP. All 13 were referred to start treatment within 24 hours.

Conclusion The AHI campaign succeeded in engaging a large number of MSM. The high numbers that completed the riskassessment tool points at an in-depth engagement with the intervention's material. While most men online were not found to be at risk for AHI, their engagement with the intervention could assist them to self-identify AHI in the future. The mix of referral sources among those diagnosed with AHI points at the added value of establishing a dedicated AHI testing service to attract risk cases in the community.
P4.106 INTENTION TO RE-TEST FOR STI AS A PREDICTOR FOR STI

Van Liere Gafs, Nicole Dukers-Muijrers, Christian Hoebe. Public Health Service South Limburg, Medical Microbiology Maastricht University Medical Centre, Geleen, The Netherlands

10.1136/sextrans-2017-053264.601

Introduction Guidelines advocate re-testing Chlamydia trachomatis(CT) and Neisseria gonorrhoeae(NG) positives within 312 months. Sexual risk behaviour is associated with CT/NG. Having a high intention to re-test could be a predictor for behaviour such as sexual risk behaviour. In this study we assess whether intention for re-testing before patients have received their test result is associated with CT or NG test result.

Methods Between 2014-2015, all STI clinic attendees answered the 5 point scale question "are you planning to return for testing the next year?" before CT/NG testing. Intention was categorised into very low(1), low(2), neutral(3) high(4) and very high(5). Patients were categorised in; women, heterosexual men and men who had sex with men in the past 6 months (MSM). The association between intention and CT/ NG was assessed using backward logistic regression with determinants; age, warned by (ex)partner, symptoms and number of sex partners $<6$ months. Neutral intention was the reference category.

Results Intention to re-test was lower among women and heterosexual men (median 3, inter quartile range (IQR) 2-4) compared to MSM (5, IQR 4-5) (both $\mathrm{p}<0.001)$. Overall CT prevalence was $12.9 \%(n=359)$ in women, $13.7 \%(n=216)$ in heterosexual men and $10.5 \%(n=116)$ in MSM. For NG this was $1.5 \%(n=43), 1.8 \%(n=28)$ and $12.1 \% \quad(n=134)$ respectively. In heterosexual men, having a high intention to re-test was associated with genital CT (OR1.5, 95\% CI 1.03-1.3). In women, having a very high intention was associated with genital NG (OR3.5, 95\% CI 1.3-9.0) and in MSM with anorectal NG (OR4.0, 95\% CI 1.4-11.7). Of CT positives, 48.9\% $(n=338)$ had (very) high intention, for NG positives this was $81.5 \%(\mathrm{n}=167)$.

Conclusion High intention to re-test was associated with genital CT/NG in heterosexuals and with anorectal NG in MSM. Healthcare providers could ask for and increase patients' intention to re-test during the consultation by motivational interviewing, even without the $\mathrm{CT} / \mathrm{NG}$ test result. Additionally re-testing should be promoted in diagnosed CT/NG positives, in accordance with guidelines.

\section{P4.107 HIV POST-EXPOSURE PROPHYLAXIS: PERCEPTION OF YOUNG STUDENTS FROM A UNIVERSITY OF BAHIA}

${ }^{1}$ Vinicius Nascimento dos Santos, ${ }^{2}$ Eveline Xavier, ${ }^{2}$ Maiara Timbó, ${ }^{1}$ Ana Gabriela Travassos. ${ }^{1}$ Universidade do Estado da Bahia, Salvador - BA, Brazili, ${ }^{2}$ Universidade Federal da Bahia, Salvador - BA, Brazil

\subsection{6/sextrans-2017-053264.602}

Introduction Post-Exposure Prophylaxis (PEP) is the use of antiretroviral treatment (ART) to reduce the risk of HIV infection after exposure. Interventions based on ART are recommended mostly for key populations, such as people aged 15 
to 24 years. This study aims to evaluate the knowledge regarding PEP amongst young university students.

Methods Cross section study carried out from August to September 2016 among Health, Education and Exact Sciences students at the State University of Bahia. Socio-epidemiological data regarding sexuality and PEP were collected through a standardised self-applied questionnaire. SPSS v20.0 was used for statistical analysis.

Results We enrolled 362 students, 85\% (305/359) females, mean age of $22.9( \pm 4.8)$ years, 83.1\% (301/362) self-declared non-white, 90.6\% (327/361) single and 43.4\% (154/355) had an income $\leq 2$ minimum wages. As for sexual behaviour, $12.1 \%(19 / 177)$ have sexual intercourse with people of the same sex, 22.6\% (63/279) have anal sex, 69.2\% (193/279) oral sex; $34.9 \%(96 / 275)$ irregular condom use, including $36.2 \%(81 / 224)$ of Health students $(p=0.36)$. As for the PEP, $39.3 \%(142 / 361)$ have heard about it, $73.6 \%(14 / 19)$ of the men who have sex with men had some knowledge $(\mathrm{p}<0.01$; OR 4.73, 95\% CI 1.67-13.45); 92.0\% (332/361) did not know the time limit to start the PEP, 93.1\% (335/360) did not know the duration and $68.9 \%(248 / 360)$ are unaware of the basic indication criteria; 87.0\% (315/360) did not know the sites for administration, $0.6 \%$ (2/361) affirm to have used it and $98.3 \%(348 / 354)$ state to not change the sexual behaviour after PEP, some said they would begin unprotected sexual practices, of which, $83.3 \%(5 / 6)$ were from the Health courses.

Conclusion The results evoke the challenges regarding the implantation of new strategies for HIV prevention. The limited knowledge regarding intervention and sites that offers PEP evidences barriers to access and prevention of new infections. These findings highlights the need for disseminate knowledge on PEP among young people, thus making it effective as an HIV prevention strategy.

\section{P4.108 INCREASING HIV TESTING OF EMERGENCY DEPARTMENT (ED) PATIENTS WITH INCREASED RISK FOR HIV AND THEIR PARTNERS AND PEERS THROUGH PROVISION OF HOME HIV SELF-TESTING KITS AND LINKAGE TO INTERNET-BASED RECRUITMENT FOR HIV TESTING - A PILOT RANDOMIZATION STUDY OF A NOVEL ED INTERVENTION}

Yu-Hsiang Hsieh, Anuj V Patel, Richard E Rothman, Angie S Kim, Mary Jett-Goheen, Danielle Signer, Carl A Latkin, Charlotte A Gaydos. Johns Hopkins University, Baltimore, USA

\subsection{6/sextrans-2017-053264.603}

Introduction Many emergency department (ED) patients with significant risks for HIV accept testing when offered in the ED but otherwise do not test for HIV on a regular basis. EDs could potentially serve as a portal for increasing HIV testing for this population beyond the ED. We conducted a pilot study to determine the acceptability of home HIV self-testing (HIVST) after the ED visit.

Methods In 2016, a pilot randomised study was conducted in an urban ED in Baltimore where an HIV screening program operated. Patients who accepted ED testing and were identified as having increased risk for HIV were enrolled. Consented patients were randomised to the HIVST group (received an oral fluid HIV home test kit), or control group (received a pamphlet regarding the importance of regular HIV testing). Participants in the HIVST group were encouraged to report self-testing results using an established online HIV/STI screening website, "IWantTheKit (IWTK)". Enrollees in the HIVST group also received 5 referral cards for their partners/ peers to request HIV self-testing kit from IWTK. Phone follow-up was conducted at 1 and 3 months. Increase in HIV testing proportion was estimated as a rate ratio (RR) using a chi-square test.

Results Overall, 100 patients were enrolled and randomised. At 3 month follow-up, 20 (40\%) patients in the HIVST group reported testing for HIV vs. $7(14 \%)$ in the control group [RR: 2.9 (95\% CI: $1.3,6.1)$ or $3.2(1.6,6.6)$ excluding 30 patients lost to follow-up]. $95 \%$ of patients in the HIVST group who reported testing for HIV used the provided kit. 9 of $19(47 \%)$ patients who self-tested at home reported their results to IWTK. None reported a reactive result for HIV. $54 \%$ of enrollees in the HIVST group reported that they distributed referral cards to their partners/peers; 6 used the referral card to request HIV and/or STI testing kits.

Conclusion This novel approach to providing HIVST kits for home testing to ED patients could increase overall HIV testing rates for patients who are at high risk, but not regularly tested, and for their partners and peers.

\section{P4.109 NARCOTICS ARE CHEAPER THAN FOOD IN PAKISTAN: OUR PWIDS ARE DYING FROM AIDS}

${ }^{1}$ Zeeshan Ayyaz, ${ }^{2}$ Kate Dolan. ${ }^{1}$ Amitiel Welfare Society, Bahawalpur - Pakistan; ${ }^{2}$ University of New South Wales, Program of International Research and Training, NDARC, Sydney, Australia

\subsection{6/sextrans-2017-053264.604}

Introduction It is estimated that worldwide there are nearly 12.7 million people who inject drugs. The UNODC estimates that Pakistan has 6.7 million drug users, of whom 4 million are addicts, giving us one of the highest number in the world. The UNAIDS Pakistan estimates 100000 people are living with HIV.

Method Our Society provides NSP, condoms, medical care and is linked to Government Victoria Hospital for rehabilitation PWIDs in Bahawalpur, Pakistan.

Results In 2014, we visited 6 drug hotspots and recruited 60 PWIDs. We examined their drug use, sexual risk behaviour, attitudes, HCV and HIV testing experience. Most were males $(80 \%)$ with a mean age of 30 years (R: 15-54 year), Muslim (92\%) and illiterate (55\%). All were local Bahawalpur PWIDs, many slept on the streets (83\%) and their source of income was scavenging from garbage (88\%). PWIDs thought their parents $(53 \%)$ and the community $(56 \%)$ hated them. They first used drugs at a mean age 18 years (R: 15-54 years). Most injected Morphine tablets, Pheniramine and Diazepam liquid (90\%), many shared syringes (85\%) and want to quit drugs $(66 \%)$. They had stolen to buy drugs $(76.7 \%)$ and been arrested (26.7\%). Most have had sexual intercourse (95\%), with sex workers (38\%), had a sexual preference for females (73\%), but few used condoms (18\%) or knew about safe sex $(21 \%)$, or STIs $(33 \%)$. Over half had genital itch $(58 \%)$. Disturbingly few PWIDs knew about HCV (10\%), just one person had been tested for HCV (1.7\%), yet many were interested in being tested for HCV (85\%). Most had been tested for HIV (73\%) and many were living with HIV (52\%). Conclusion Pakistani PWIDs suffer many problems, lack access to harm reduction services and are dying from inaction. We 